\title{
COMMONWEALTH PANDEMIC PREPAREDNESS PLANS
}

John Horvath AO

Chief Medical Officer

Commonwealth Department of Health and Ageing

\section{ABSTRACT}

This paper describes the work being done by the Australian Government Department of Health and Ageing to prepare for a possible influenza pandemic. It provides an overview of Australian Government initiatives and explains both the purpose and the content of the Australian Health Management Plan for Pandemic Influenza. It summarises efforts to improve regional detection and response capabilities and it explains the role of simulation exercises in Australian pandemic planning.

The continuing global outbreak of H5N1 avian influenza (hereafter also referred to as $\mathrm{H} 5 \mathrm{~N} 1$ ) has raised the spectre of a new influenza pandemic. In H5N1, we have a novel influenza strain that is highly pathogenic to many bird species and which has crossed the species barrier to cause severe disease in humans and other mammals. Thankfully, H5N1 does not easily infect humans. At the time of writing there have only been a few more than 240 cases worldwide, despite large numbers of people living in close proximity to infected birds. Nor does it pass efficiently between humans, as our seasonal human influenza strains do. While it is not inevitable that $\mathrm{H} 5 \mathrm{~N} 1$ will take the next step to become an effective human pathogen and so trigger a pandemic, its existence provides a timely reminder that all nations individually and collectively need to be fully prepared for the next influenza pandemic, whatever its origin.

Australia is well prepared to respond to an outbreak of avian influenza or an influenza pandemic, thanks to the years of hard work by government departments, agencies, institutions and individuals across the country. This article briefly describes the range of initiatives undertaken by the Australian Government Department of Health and Ageing to prepare Australia and the region for a possible influenza pandemic. It provides an overview of the Australian Health Management Plan for Pandemic Influenza. ${ }^{1}$ It also provides an update on arrangements for a national pandemic management simulation exercise to be held in October 2006.

\section{OVERVIEW OF COMMONWEALTH INITIATIVES}

The Australian Government has devoted significant resources to building national preparedness and a capacity for an immediate and effective response to any pandemic alert. These measures include:

- creating an Office of Health Protection to develop and coordinate Australia's response to health threats and emergencies such as pandemic influenza

- establishing the National Medical Stockpile, which has one of the largest per capita supplies of influenza antivirals in the world as well as personal protective equipment and other essential health supplies to deploy during an influenza pandemic

- strengthening Australia's communicable disease surveillance networks and laboratory capacity

- contracting two influenza vaccine manufacturers for a guaranteed pandemic vaccine supply to protect the Australian population

- funding CSL Limited (a pharmaceutical company) to fast track production of an $\mathrm{H} 5 \mathrm{~N} 1$ candidate vaccine to allow for more rapid production of a pandemic vaccine should the pandemic strain be closely related to $\mathrm{H} 5 \mathrm{~N} 1$

- providing substantial research grants to help solve some of the basic questions around pandemic influenza

- providing technical and financial assistance to several of our regional neighbours to strengthen their human and animal communicable disease control systems and pandemic preparedness planning

- developing a comprehensive communications strategy for use in the event of a pandemic

- conducting a pandemic simulation exercise in late 2006

- funding an upgrade of the World Health Organization Collaborating Centre for Reference and Research on Influenza in Melbourne.

More information on these and other initiatives and on the role of the Office of Health Protection can be found at www.health.gov.au.

\section{THE AUSTRALIAN HEALTH MANAGEMENT PLAN FOR PANDEMIC INFLUENZA}

The 2006 Australian Health Management Plan for Pandemic Influenza (the 'Plan'), building on earlier pandemic plans, provides an overarching guide for the Australian health response to a pandemic influenza threat. The updated Plan is an accessible, plain English document designed to meet the information needs of the widest range of Australians.

The Plan is complemented by a series of technical papers. Some of these papers-for example, Interim Clinical Guidelines $^{2}$ and Interim Infection Control Guidelines ${ }^{3}$ - are available now. Others will be released progressively in the coming months. The Plan and copies of currently available technical papers are available from www.health.gov.au.

Underpinning the Plan is an enhanced understanding of the likely public health responses to an outbreak of pandemic influenza. In December 2005, a report was commissioned by the Australian Government Department of Health and Ageing to use mathematical models to assess public health responses to an outbreak of infectious viral respiratory disease. $^{4}$

The resultant modelling points to the benefit of early detection and rapid response and to a synergistic effect of 
public health interventions supporting the use of multiple strategies. It indicates that a containment strategy is likely to be effective and may contain a pandemic for several months. It further indicates that community public health interventions such as social distancing and home quarantine, together with the use of targeted antiviral prophylaxis for people exposed to the virus, can dramatically reduce transmission. It indicates that containment of a pandemic may be achieved if intensive multiple public health efforts are applied.

The Plan is complemented by the National Action Plan for a Human Influenza Pandemic (NAP), which brings together Commonwealth and state and territory government planning. The NAP was prepared by the Council of Australian Governments and is available from www.pmc. gov.au.

\section{REGIONAL AND INTERNATIONAL INITIATIVES}

The Australian Government has committed \$152 million to initiatives to improve the detection and surveillance, emergency preparedness and response capabilities of countries in the region.

In November 2005, the Prime Minister announced funding of $\$ 100$ million over four years for initiatives that will combat the threat of pandemics and other emerging infectious diseases within the Asia-Pacific region. Some $\$ 90$ million will be used to help regional economies prepare and to support organisations working across the region, while the remaining $\$ 10$ million will be used for specific Asia-Pacific Economic Cooperation (APEC) activities on avian influenza.

Australia is working closely with peak multilateral agencies such as World Health Organization, the Food and Agriculture Organization, and the World Animal Health Organization. It also works closely with regional organisations such as the Association of Southeast Asian Nations and the APEC forum, and with other major donor countries and agencies to coordinate assistance for avian and pandemic influenza in the region. Australia participated in the International Pledging Conference on Avian and Human Influenza in Beijing in January this year and we have been working closely with our regional partners to ensure that assistance is effectively provided to countries in need, without duplication of effort.

Work has also been done at the international level to ensure an effective global response to avian and pandemic influenza. Australia is a foundation member of the International Partnership on Avian and Pandemic Influenza, which brings together concerned states to develop our global capabilities to respond to the pandemic threat in a coordinated manner. This International Partnership first met in October 2005 and met again in June 2006.

Australia is also coordinating a range of regional pandemic projects, including an APEC pandemic communications exercise that aims to maximise regional coordination of pandemic influenza responses. An APEC project on 'Functioning Economies During Times of Pandemic' will assist in ensuring economic life can continue in the event of a pandemic.

\section{SIMULATION EXERCISES}

The Australian Government recognises the importance of testing our preparedness for health disasters such as influenza pandemics. One of the most effective ways to test preparedness and response plans is by conducting exercises.

In late 2005, all states and territories participated in Exercise Eleusis 05, an avian influenza outbreak desktop exercise conducted under the auspices of the Australian Government Department of Agriculture, Fisheries and Forestry. The aim of the exercise was to evaluate Australia's capability to manage emergency zoonotic disease outbreaks across industry and government, including testing: (a) the integration of nation-wide emergency zoonosis arrangements, (b) communication capabilities and (c) disease control policies and strategies.

Specific objectives for Australian Government Department of Health and Ageing in relation to Exercise Eleusis included evaluating performance and compliance in relation to the 2005 Management Plan, communications between participating agencies and with key advisory committees, and governance arrangements of actions such as antiviral deployment, medical equipment deployment and potential activation of quarantine. Surveillance arrangements, media communications, and information sharing between agencies were also evaluated.

Lessons learned from Eleusis are helping the Australian Department of Health and Ageing to conduct a national influenza pandemic exercise to assess the Australian human health system's capacity to effectively respond to an influenza pandemic. This exercise, Exercise Cumpston 06, will focus on the Australian health response as set out in the 2006 Australian Health Management Plan for Pandemic Influenza and related technical papers. It will test Australia's level of preparedness and identify any gaps or shortfalls. The exercise is named after John Howard Lidgett Cumpston, the first Director-General of the Commonwealth Department of Health, who was appointed when it was created in 1921. Cumpston was in charge of the Quarantine Service in 1918 and is credited with introducing Australia's successful influenza quarantine measures.

The live simulation phase of the exercise will occur in October 2006 and will test everything from border measures to localised outbreak containment and public health responses. It will exercise key decision-making steps and processes to move to the maintenance phase during a pandemic. While the live exercise will be conducted in one state, all the other states and territories will be involved, enabling the Government to test governance arrangements and strategic level decision-making within and between jurisdictions at all levels. 
Other elements of the Plan have been covered through discussion exercises and workshops in the months leading up to the operational phase. These activities have addressed border control and screening, decision-making, deployment of the national medical stockpile and the national health emergency response. They have involved all states and territories, as well as Australian Government agencies.

Effective communication between participants and between participants and the public/front-line health providers is a particular focus of all elements of Cumpston 06. A final consolidated report will be prepared in early 2007 to disseminate the findings to policy makers and to enable future exercises to draw on the Cumpston 06 experience.

\section{CONCLUSION}

The Australian Government has set in train a wide range of initiatives to prepare the nation in the event of an influenza pandemic. Planning and plan testing, involving all levels of Government, are important parts of the work being done to limit the impact of any pandemic on the well being of Australians.

\section{REFERENCES}

1. Department of Health and Ageing. Australian health management plan for pandemic influenza. Canberra: Department of Health and Ageing, 2006.

2. Department of Health and Ageing. Interim national pandemic influenza clinical guidelines. Annex to Australian health management plan for pandemic influenza. Canberra: Department of Health and Ageing, 2006.

3. Department of Health and Ageing. Interim infection control guidelines for pandemic influenza in healthcare and community settings. Annex to Australian health management plan for pandemic influenza. Canberra: Department of Health and Ageing, 2006.

4. Becker N, Glass K, Barnes B, Caley P, Philp D, McCaw et al. Using mathematical models to assess responses to an outbreak of an emerged viral respiratory disease. Canberra, Australian Government Department of Health and Ageing: unpublished research paper, 2006. 암

\section{PREPARING FOR THE NEXT INFLUENZA PANDEMIC: A NEW SOUTH WALES PERSPECTIVE}

\section{Paul Armstrong}

Biopreparedness Unit

NSW Department of Health

\section{Kerry Chant \\ Centre for Health Protection \\ NSW Department of Health}

\section{ABSTRACT}

Pandemic influenza is one of a small number of infectious diseases that pose a significant global threat. Pandemic preparedness has accelerated around the world in recent years in response to the perceived increased risk of a pandemic developing following the emergence of $\mathrm{H} 5 \mathrm{~N} 1$ avian influenza in domestic poultry flocks in Asia, Africa and Europe. There is a hierarchy of pandemic plans international, national, state, and local - and harmonisation of all of these is imperative for a coordinated and effective response. At the national and state levels, plans have been developed for a whole-of-government response to a pandemic, in addition to plans specifically for the health sector. It is inevitable that influenza pandemics will occur and careful planning is crucial to mitigate their potentially devastating effects.

Pandemic influenza is one of a small number of infectious diseases that pose a significant global threat. This group of infectious diseases are characterised by a capacity to cause significant degrees of illness, death, economic loss, and public fear and panic. A convenient way of classifying them is as emerging, re-emerging, and deliberately emerging infectious diseases. ${ }^{1}$ Emerging infectious diseases are those that have never been recognised before, a recent example being severe acute respiratory syndrome (SARS). Influenza pandemics are an example of re-emerging infectious diseases, which are those that have been recognised for some time but undergo a resurgence in a different form or geographical location. Deliberately emerging diseases are those that are intentionally introduced and are synonymous with diseases caused by agents of bioterrorism.

These different types of infectious diseases are dealt with here as a group because there are many overlapping preparedness activities for each of them. ${ }^{1}$ Of these disease threats, influenza pandemics present the greatest potential risk in that, by their very nature, the entire population will be at risk at the same time and the virus spreads quickly. Should the pandemic influenza virus be highly virulent, as H1N1 was in 1918-1919, then large-scale deaths could result. SARS, by comparison, caused less than 0.1 per cent of the deaths that resulted from the mildest influenza pandemic of the last century. ${ }^{2,3}$ The magnitude of the threat to the public health posed by an influenza pandemic means that preparing adequately for such an event will go a long way towards preparing for all large-scale infectious disease threats.

\section{WHY DO WE NEED TO PREPARE?}

The world needs to prepare for the next influenza pandemic for two important reasons. First, a pandemic will occur at 